\title{
Cefaleas en el escolar
}

\author{
DRES.: RICARDO GUZMAN C. *, RICARDO GUZMAN Y. *, ERIC SAELZER W. **.
}

El problema de la cefalea como entidad clínica es frecuente en la infancia. (1-2-3-5-9).

Las estadísticas al respecto son dispares y van, desde Bille - 1962- que estudió 9.000 escolares suecos encontrando que el $75 \%$ de ellos había padecido algún tipo de cefaleas, hasta Bergman -1963- que dice que el 1\% de los niños en U.S.A. habían presentado dolor de cabeza que los llevó a consultar médico.

En Chile no conocemos la prevalencia del problema en la población escolar ya que no encontramos investigaciones al respecto.

Esperamos que nuestro trabajo aporte algunos antecedentes para el estudio de la Cefalea del escolar, cuando ella se presenta como síntoma principal y recidivante, así como los cuadros clínicos que la producen, con el propósito de clarificar un problema tan escasamente estudiado (5-10).

Material y metodo. Se tomó un colectivo de 45 niños con cefalea pertinaz y persistente para un estudio prospectivo de 6 meses de duración. Los casos fueron enviados al Depto. de Cefaleas desde cuatro Consultorios Periféricos representativos de los distintos niveles socioeconómicos de la población urbana del Area Occidente, Santiago.

Se elaboró una ficha individual que contemplaba el registro de:

- Antecedentes del paciente: edad, sexo, escolaridad y rendimiento escolar.

\footnotetext{
* Depto. Cefaleas, Servlcio de Medicina Hospital San Juan de Díos.

** Servicio de Pediatria, Hospital San Juan de Dios.
}

- Antecedentes mórbidos del grupo familiar.

- Estudio de las crisis: edad de inicio, factores antecedentes; síntomas acompañantes; localización, duración, intensidad, frecuencia y carácter del dolor.

- Exámenes clínicos: Físico general, Neurológico, Otorrinológico, Oftalmológico y E. E. G., en todos los casos. Se solicitó radiografía de cráneo sólo en seis pacientes en que se estimó necesario.

Quedaron fuera del estudio final tres casos en los que, por algún motivo, no pudo realizarse el estudio completo.

- Dada la imposibilidad de conocer el "universo" de escolares con cefaleas del cual extraer una "muestra representativa" cuyos resultados generalizar al mismo universo, se tomó un grupo de niños que constituyeron un "colectivo". Todos los resultados de su estudio " pueden aplicarse para "grupos similares" de escolares con cefaleas. Aún más, dado que estos resultados se analizaron utilizando medidas estadísticas y probabilísticas no paramétricas, tales como $\mathrm{x}^{2}$, las conclusiones que indicamos como estadísticamente significativas, pueden generalizarse a cualquier grupo de escolares con cefalea.

ANALISIS DE los RESUlTADOS. Los escolares que consultaron por Cefalea fluctuaban entre los cinco y catorce años de edad. Del

\footnotetext{
* Los datos se analizaron en la computadora Cannon 1614 P, Universidad de Chile, Sede Sur.
} 
TABLA № 1

DISTRIBUCION DE 45 ESCOLARES CON CEFALEA, SEGUN EDAD Y SEXO

SANTIAGO, 1975

\begin{tabular}{rccc}
\hline Edad & Hombres & Mujeres & Total \\
\hline $5-10$ & 9 & 15 & 24 \\
$11-14$ & 9 & 12 & 21 \\
\hline TOTAL & 18 & 27 & 45 \\
\hline
\end{tabular}

TABLA No 2

EDAD DE INICIO DE CRISIS DOLOROSAS EN 45 ESCOLARES CON CEFALEA

SANTIAGO, 1975

\begin{tabular}{rcc}
\hline \multicolumn{1}{c}{ Años } & No Casos & $\%$ \\
\hline $2-3$ & 6 & 13,4 \\
$4-6$ & 18 & 40.0 \\
$7-9$ & 8 & 17.7 \\
$10-12$ & 13 & 28.9 \\
$12-15$ & - & - \\
\hline TOTAL & 45 & 100.0 \\
\hline
\end{tabular}

total, dieciocho eran hombres y veintisiete mujeres. Al momento de la consulta, la escolaridad iba desde Kinder a $2^{\circ}$ Medio. (Tabla No 1).

La diferencia de proporción entre varones y niñas $(z=1,94)$ no sería significativa en cuanto a presentar cefaleas. La escolaridad tampoco sería significante.

En cuanto al rendimiento escolar los pacientes se distribuyeron en todas las categorías, desde malo a óptimo, siguiendo la misma tendencia que se observa en el rendimiento de escolares corrientes.

Entre los antecedentes mórbidos del grupo familiar, un tercio $(33,3 \%)$ de los niños tenía uno o más de sus parientes directos jaquecosos, lo que se considera significativo. El $11,1 \%$ tenía familiares que padecían de algún tipo de epilepsia. En el grupo familiar del $8,9 \%$ de los niños encontramos antecedentes de jaqueca y epilepsia. Este mismo porcentaje correspondió a parientes con alcoholismo.

Las crisis dolorosas se habían presentado por primera vez entre los 4 y 6 años de edad, en un $40 \%$ de los casos. (La probabilidad estadística de que este resultado se deba al azar es inferior a 0,05. Esto es, $\mathrm{P}<0,05$ ). (Tabla $\mathrm{N}: 2$ y figura $\mathrm{N}^{\circ} 1$ ).

\section{EDAD DE INICIO DE CRISIS DOLOROSAS SANTIAGO, 1975}

\section{ESCOLARES}

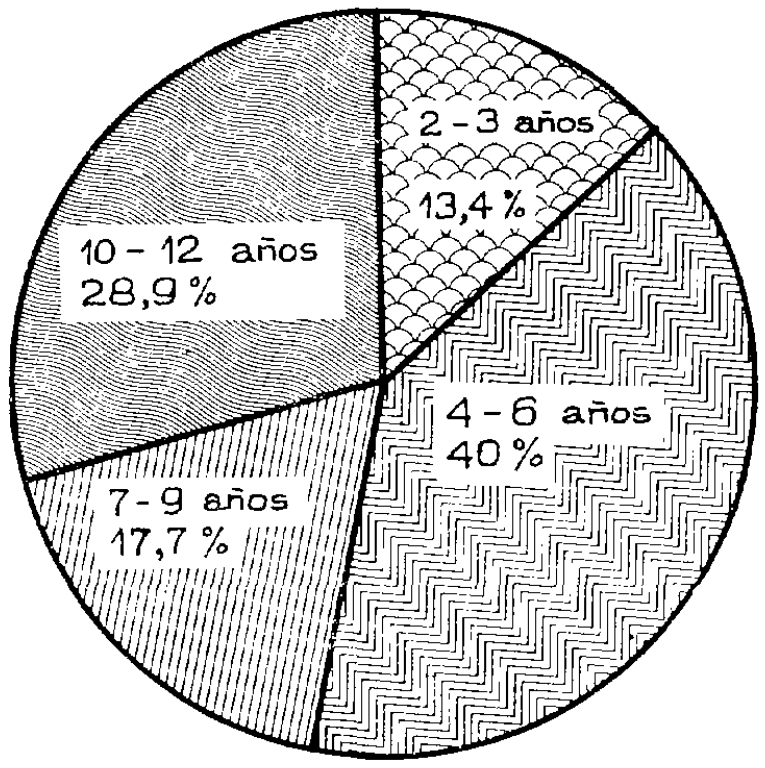

Figura No 1

En cuanto al factor antecedente a las crisis de dolor, apareció como relevante el stress en un $44,4 \%$ de los niños $(P<0,05)$.

Más de la mitad de los pacientes (60\%) presentaba otros síntomas acompañando la cefalea $(\mathrm{P}<0,05)$. Los síntomas más frecuentes eran náuseas y/o vómitos, a los que en algunos casos se agregaban anorexia, dolores abdominales y otros.

En 20 de los niños el dolor tenía una localización frontal, lo que corresponde a un porcentaje significativo. Otros 10 , tenían hemicráneas típicas. En los 15 restantes el dolor tenía otras localizaciones.

En cuanto a la duración del dolor, es altamente significativo que las crisis duraran de 2 a 6 horas en el 77,8\% de los casos; menos de una hora persistía el dolor en el $13,3 \%$; y más de 6 horas sólo en el $8,9 \%$. 
También aparece como estadísticamente significativa la frecuencia de aparición de las crisis. El dolor se presentaba en un 71,1\% de los niños con una periodicidad que iba desde diaria a semanal. Crisis con intervalos de aparición mayores a 15 días, se dieron en el $22,2 \%$ de los casos.

El dolor era de tipo pulsátil en el $40 \%$ de los niños. Los dolores de tipo opresivo y punzante le siguieron, cada uno con un $22,2 \%$ de la muestra.

En el 100\% de estos escolares, el dolor no había cedido a la terapia con analgésicos de uso habitual. La intensidad de él tenía un carácter absolutamente invalidante en el $51,1 \%$ de los niños: durante las crisis debían permanecer en cama, impidiéndoles asistir a clases. En dos de estos niños llegaba a producir llanto.

En cuanto a los resultados de los exámenes físico general y neurológico, ellos estuvieron dentro de los límites normales en todos los casos.

El examen oftalmológico reveló en unos pocos niños alteraciones tales como miopía y astigmatismo hipermetrópico, en su mayoría corregidos. Ninguna de estas patologías originaba la cefalca en consulta.

El examen otorrino reveló tres casos de rinosinusitis; en dos pacientes eran causa directa de la cefalea.

Se practicó E. E. G. en todos los casos. El $62,2 \%$ de ellos se informaron como normales. Un $24,4 \%$, con signos de actividad comicial intercrítica. El 13,3\% presentaba alteraciones inespecíficas. (Tabla $N \div 3$ y figura N.2).

De Ios 45 niños, en $19(42,2 \%)$ la Cefalea correspondió a Jaqueca. 12 casos $(26,7 \%)$ fueron diagnosticados como un equivalente convulsivo y $12(26,7 \%)$ como Cefalea psicogénica. El resto, 2 escolares $(4,4 \%)$ padecían

TABLA No 3

\section{RESULTADOS ELECTROENCEFALOGRAMA EN 45 ESCOLARES CON CEFALEA}

SANTIAGO, 1975

\begin{tabular}{lcc}
\hline \multicolumn{1}{c}{ E. E. G. } & No $^{\circ}$ Casos & $\%$ \\
\hline Normales & 28 & 62.2 \\
Disrritmia Comicial & 11 & 24.4 \\
Alteraciones Inespecificas & 6 & 13.4 \\
\hline \multicolumn{1}{c}{ TOTAL } & 45 & 100.0 \\
\hline
\end{tabular}

DIAGNOSTICO EN 45 ESCOLARES CON CEFALEA SANTIAGO, 1975

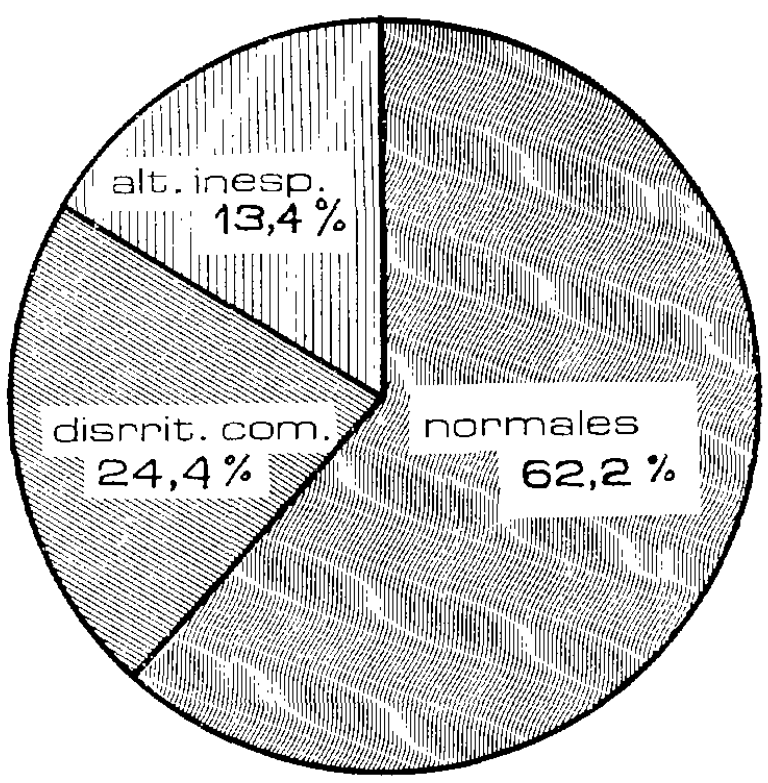

Figura $\mathrm{N}^{\circ} 2$

TABLA № 4

\section{DIAGNOSTICO EN 45 ESCOLARES QUE CONSULTAN POR CEFALEA}

SANTIAGO, 1975

\begin{tabular}{lcc}
\hline \multicolumn{1}{c}{ Diagnóstico } & No Casos & $\%$ \\
\hline Jaqueca & 19 & 42.2 \\
Equival. Convul. & 12 & 26.7 \\
Cefalea Psicogénica & 12 & 26.7 \\
Rinosinusitis crónica & 2 & 4.4 \\
\hline
\end{tabular}

de rinosinusitis; uno de ellos crónica y el otro, alérgica.

Comentario. En algunas oportunidades una consulta médica es catalogada como banal. Si el paciente es un niño que padece de cefalea, y si con el examen físico general es posible descartar un proceso infeccioso $-\mathrm{y}$, aún más, si el examen neurológico de rutina es normal- este síntoma tiende entonces a ser desestimado por el pediatra general.

Esto se explicaría porque la mayoría de los niños portadores de un cuadro de cefalea tenaz y recidivante, consulta en el período intercrítico. De allí la tardanza con que estos enfermos son enviados a centros especializados. 
RESULTADOS ELECTROENCEF ALOGRAMA

45 ESCOLARES - STGO., 1975

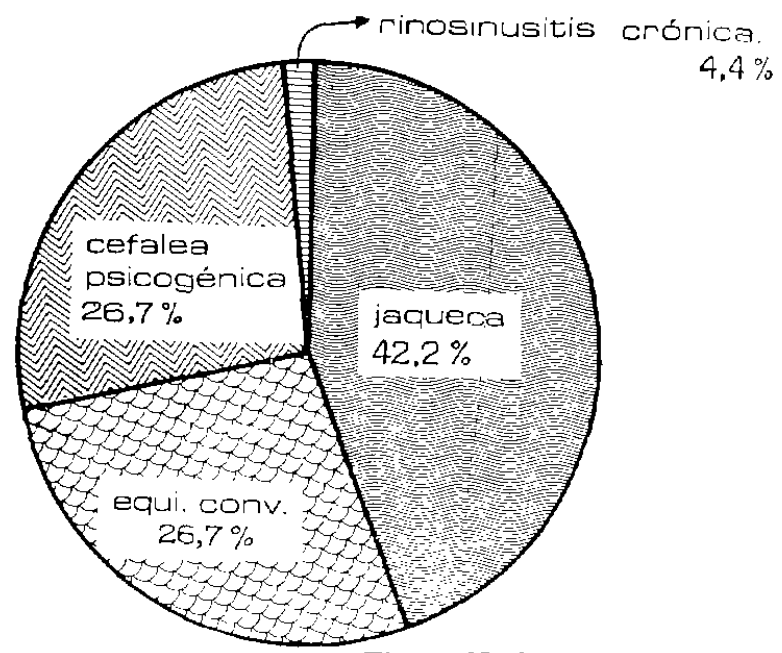

Figura No 3

Si a esto agregamos que la cefalea se inicia entre los 4 y 6 años de vida; que a esa edad los niños ya son capaces de definir la localización de su dolor $(1,2,3)$; que las crisis tienen un carácter invalidante; $\mathrm{y}$, que conllevan cronicidad, se enfatiza aún más la necesidad de llegar a un diagnóstico pre$\operatorname{coz}$ y correcto.

En la muestra que estudiamos, la jaqueca, el equivalente convulsivo y la cefalea psicogénica -cuadros que generalmente se inician en la infancia $(4,8,9)$ - constituyen los hallazgos de mayor frecuencia $(42,2 \%, 26,7 \%$ y $26,7 \%$ respectivamente). En dos pacientes la cefalea fue atribuida a rinosinusitis crónica, una de ellas de origen alérgico (tabla N: 4 y figura $N$ ㅇ 3 ).

No encontramos en esta casuística cefaleas de origen oftálmico coincidiendo con autores $(7,10)$ que hablan de la escasa frecuencia de esta etiología en la infancia.

Tampoco encontramos niños que padecieran de lesiones expansivas intracraneanas. Cabe indicar trente a esta situación que la cefalea no sería el síntoma más frecuente en este tipo de cuadros $(6,7)$ cuyas manifestaciones suelen ser atípicas, destacando las alteraciones del carácter y la aparición de compromiso neurológico. No obstante, la existencia de un tumor cerebral $\mathbf{u}$ otro tipo de lesión expansiva debe ser siempre investigada exhaustivamente en un niño con cefalea. En ese sentido, los exámenes neurológico y oftalmológico son básicos en una primera aproximación diagnóstica; la radiografía de cráneo y otros exámenes especiálizados sólo serían necesarios frente a una sospecha.
Nuestra metódica de estudio nos parece bastante útil ya que permitió delimitar la causa de las crisis de cefalea en todos nuestros pacientes.

La respuesta al tratamiento ha sido buena ( $85 \%$ asintomáticos) lo que estaría señalando el buen pronóstico de estos casos cuando se logra realizar un diagnóstico correcto.

\section{RESUMEN}

Se estudian prospectivamente 45 escolares que padecian de cefalea crónica tenaz y recidivante.

Se encuentra que la mayor frecuencia etiológica está dada por Jaqueca, Equivalente Convulsivo y Cefalea Psicogénica $(42,2 \%$, $26,7 \%$ y 26,7\%). Los dos primeros, por lo general, de iniciación en la infancia.

Se observa en este colectivo que cuadros de otra etiología, tales como los rinosinusales $(4,4 \%)$ y oftálmicos, son de escasa frecuencia.

No se detectaron procesos expansivos intracraneanos, sin embargo se recalca que esta etiología debe tenerse siempre presente y agotarse el estudio especializado frente a una sospecha.

Se sugiere la forma como deben estudiarse estos enfermos y se enfatiza el buen pronóstico si el diagnóstico es precoz.

\section{SUMMARY}

A prospective study was performed in a group of 45 school age children that suffered from chronic and recurring cephalalgia.

The etiologies most commonly found were migraine $(42,2 \%)$ convulsive equivalent $(26,7 \%)$ and psychogenic cephalalgia $(26,7 \%)$.

Other etiologies such as the rhinosinuses $(4,4 \%)$ or ofthalmic diseases are present in only a small group of patients.

No intracraneal expansive processes were detected, but emphasis is made upon the fact that this diagnosis must always be borne in mind and thoroughly discarded.

The method by which these patients must be studied is suggested insisting on the good prognosis in cases of early diagnosis.

\section{REFERENCIAS}

1.- Ajuriaguerra, J. Manual de Psychiatrie de L'enfant. Masson y Cie. Paris, 1970. 
2.-Bell, W. E. Headache in Childhood. Headache, 8: $127,1968$.

3.-Bray, P. F.: Neurology in Pediatrics. Year BoBok Medical Publ. Chicago, 1969.

4.-Devilat, M.; Napolitano, E., Cox, J. Equivalente Convulsivo: iuna forma de epilepsia? Rev. Chil. de Pediatría 95: 52, 1974.

5.-- Ferry, Peggy C. Diagnosis and Office Management of Headaches in Children. Clinical Pediatrics. 11: 195,1972 .

6.-Froelich, W. A.; Carter, C. C.; O'Leary, J.; Rosenbaum, H. Headache in Childhood. Neurology, $10 ; 639,1960$.
7.-Koch, Christian; Melchior, Johannes C. Headache in Childhood. Danish Medical Bulletin, 16: 109, 109.

8.-Poch, G.; Martín, A. Zavala, H. Cefaleas epilépticas puras. Cefaleas y Jaquecas. Eudeba, Bs. As., 1973.

9.-Ryan, R. E. Migraine in Children. Headache, 8: 67, 1968.

10.-Zanjanian, M. H. A Review of Headaches in Children. The Journal of the Medical Society of the Medical Society of New Yersey. 71: 755, 1974.

\section{AGRADECIMIENTOS}

Agradecemos a la señorita Teresa Segure, Sede Sur Unlversidad de Chile, por su valiosa colaboración en la interpretación estadística de los datos obtenidos. 